\title{
Tapping with intentional drift
}

\author{
A. N. Vardy $\cdot$ A. Daffertshofer $\cdot$ P. J. Beek
}

Received: 21 December 2007/ Accepted: 1 September 2008/Published online: 2 October 2008

(C) The Author(s) 2008. This article is published with open access at Springerlink.com

\begin{abstract}
When tapping a desired frequency, subjects tend to drift away from this target frequency. This compromises the estimate of the correlation between inter-tap intervals (ITIs) as predicted by the two-level model of Wing and Kristofferson which consists of an internal timer ('clock') and motor delays. Whereas previous studies on the timing of rhythmic tapping attempted to eliminate drift, we compared the production of three constant frequencies $(1.5,2.0$, and $2.5 \mathrm{~Hz})$ to the production of tapping sequences with a linearly decreasing inter-tap interval (ITI) (corresponding to an increase in tapping frequency from 1.5 to $2.5 \mathrm{~Hz}$ ). For all conditions a synchronization-continuation paradigm was used. Tapping forces and electromyograms of the index-finger flexor and extensor were recorded and ITIs were derived yielding interval variability and model parameters, i.e., clock and motor variances. Electromyographic recordings served to study the influence of tapping frequency on the peripheral part of the tap event. The condition with an increasing frequency was more difficult to perform, as evidenced by an increase in deviation from the intended ITIs. In general, tapping frequency affected force level, inter-tap variability, model parameters, and muscle co-activation. Parameters for the condition with a decreasing ITI were comparable to those found in the constant frequency conditions. That is, although tapping with an intentional drift is different from constant tapping and more difficult to perform, the timing
\end{abstract}

A. N. Vardy $(\varangle)$ · A. Daffertshofer · P. J. Beek

Research Institute MOVE, Faculty of Human Movement

Sciences, VU University Amsterdam, Van der Boechorststraat 9,

1081 BT Amsterdam, The Netherlands

e-mail: a.vardy@fbw.vu.nl

URL: http://www.fbw.vu.nl/ avardy/ properties of both forms of tapping are remarkably similar and described well by the Wing and Kristofferson model.

Keywords Neural timer $\cdot$ EMG $\cdot$ Motor control · Error correction · Drift

\section{Introduction}

The search for timing mechanisms underlying the production of repetitive movements has a long tradition dating back as far as the late nineteenth century (Stevens 1886). Rhythmic finger tapping has received much interest from researchers of motor control because it provides an expedient window into neural timing. Although many brain areas participate in the timing of motor behaviorincluding the supplementary motor area (Grahn and Brett 2007), cerebellum (Ivry et al. 1988; Ivry 1997; Del Olmo et al. 2007), basal ganglia, thalamus, and motor and sensory cortices (Ivry and Richardson 2002) - the notion of a single, abstractly defined, neural timer has proven rather successful in accounting for behavioral data.

A case in point is the two-level model proposed by Wing and Kristofferson (1973)-here referred to as the WKmodel-to account for the serial correlations between successive time intervals. The model consists of a clock, defining the duration of the interval between two neural commands, and motor delays, which represent the time it takes for a neural command to reach the end-effector, i.e., the lag at which the tap occurs. The model does not require any assumptions regarding the origin of the clock, only that the timing intervals are independent. Since its inception, the WK-model has been frequently used to analyze tapping sequences in a so-called synchronization-continuation paradigm, in which subjects first synchronize their tapping 
to a metronome and then continue the previously indicated rhythm after removal of the metronome (Wing and Kristofferson 1973; Musha et al. 1985; Vorberg and Wing 1996; Pressing and Jolley-Rogers 1997; Pressing 1998; Ivry and Richardson 2002). The elegantly simple WKmodel provides an adequate description of various statistical properties of tapping sequences during continuation that can stand up to more elaborate models, which typically place more emphasis on long-term correlations in voluntary tapping (e.g., Chen et al. 1997; Daffertshofer 1998; Delignières et al. 2004).

In this study, we examine the generality of the WKmodel by manipulating task difficulty using motor performances with changing tempo. We analyze the temporal properties of inter-tap interval (ITI) sequences in terms of their variance, autocorrelation, and WK-model parameters, for isochronous and non-isochronous tasks. Pilot studies indicated that a non-isochronous task was more difficult to perform than an isochronous one. Task difficulty affects performance in a number of ways. On a behavioral level, mental load influences biomechanical properties of rhythmic finger movements (Loehr and Palmer 2007) and more difficult tasks (tapping a rhythm) display more variability (Doumas and Wing 2007). Furthermore, cortical areas may be activated differently during simple and difficult tasks. Gerloff et al. (1998) showed that perturbations of the primary motor area using transcranial magnetic stimulation resulted in more performance errors during complex tasks than during simple tasks. Different cortical areas reveal different interactions as a result of task difficulty (Manganotti et al. 1998), and the temporal structure of neural activity is generally influenced by increased mental load (Dhamala et al. 2002).

In isochronous tapping subjects deviate from the fixed target tapping frequency by means of long-term drift (Vorberg and Wing 1996). This unintentional phenomenon has proven detrimental to the fit of the WK-model as it may either result in corrections that yield long-term correlations (e.g., Kaulakys 1999; Ding et al. 2002) or, when monotonic, in non-stationary time series causing improper estimates of the autocorrelation function and ultimately in a poor model fit. A sharp distinction between long-term properties and non-stationarity is difficult to make since unintentional drift is hard to control experimentally. In practice, many experimenters keep time series as short as possible to minimize the influence of drift. ${ }^{1}$ Instead of regarding drift as a confounding factor, however, it may be studied in its own right and explicitly incorporated in the experimental design. By introducing intentional drift, drift

\footnotetext{
${ }^{1}$ More recently, though, efforts have been made to analyze (Madison 2001) and to model (Collier and Ogden 2004) unintentional deviations from isochronous tapping.
}

may become steady and hence predictable enough to be corrected by linear detrending (Vorberg and Wing 1996). Such an approach has been used to study synchronization error (Schulze et al. 2005) and subjects' ability to sustain drift during the continuation phase of a synchronizationcontinuation paradigm (Madison and Merker 2005). To date, however, intentional drift has not been analyzed against the background of the WK-model. Here, we apply intentional drift to study temporal properties of tapping a non-constant sequence.

Does intentional drift modify task difficulty and does this mean that the WK-model fails to describe the behavioral data? An inaccurate description may be caused by the emergence of temporal correlations in the presence of intentional drift. Such correlations may imply that other mechanisms become involved, reflecting the large number of brain areas that are active in neural timing. Indeed, a change in tempo can have profound influences on the temporal properties of motor performance even if the change is constant. ${ }^{2}$ In general, tapping a sequence with increasing or decreasing tempo cannot be continued forever, unlike tapping at a constant pace (attentional and energetic limitations aside). Anticipating the up-coming saturation in pace may require additional planning, at least to some degree, making the intentional drift task more difficult, in turn affecting the temporal structure of the sequence preceding saturation. To anticipate, for our subjects (non-musicians), intentional drift sequences did impose an additional burden on performance. However, performance remained adequate so that, after linear detrending, we could analyze temporal properties of intentional drift sequences similar to tapping at a constant pace, that is, in terms of a timer and its autocorrelations. Put differently, the WK-model remained applicable to tapping with a constant, intentional drift. In addition, we estimated motor delay variability in the WK model and its tempo dependence using electromyograms (EMGs) of the finger flexor and extensor muscles. The combination of the flexor and extensor EMG was used to calculate a measure of muscle co-activation, which may influence the motor delay.

\section{The WK-model}

The WK-model is depicted schematically in Fig. 1. In brief, the model supposes that inter-tap intervals (ITIs) arise from clock intervals $C_{i}$ and motor delays $D_{i}$. Both sequences are assumed to be independent in time and independent from each other. Under this assumption, the

\footnotetext{
${ }^{2}$ As sketched in the next section, the WK-model can be readily extended to accommodate linear drift by modifying the neural clock; this keeps the correlation structure of the detrended ITIs intact.
} 
Fig. 1 The two-level timer model proposed by Wing and Kristofferson: $C_{i}$ denote clock intervals (mean $\mu_{\mathrm{C}}$, variance $\sigma_{\mathrm{C}}^{2}$ ) and $D_{i}$ denote motor delays (mean $\mu_{\mathrm{D}}$, variance $\sigma_{\mathrm{D}}^{2}$ ); intertap intervals (ITIs) are defined as $I_{i}=C_{i}+D_{i+1}-D_{i}$ (mean $\mu_{\mathrm{C}}$, variance $\left.\sigma_{\mathrm{C}}^{2}+\sigma_{\mathrm{D}}^{2}\right)$

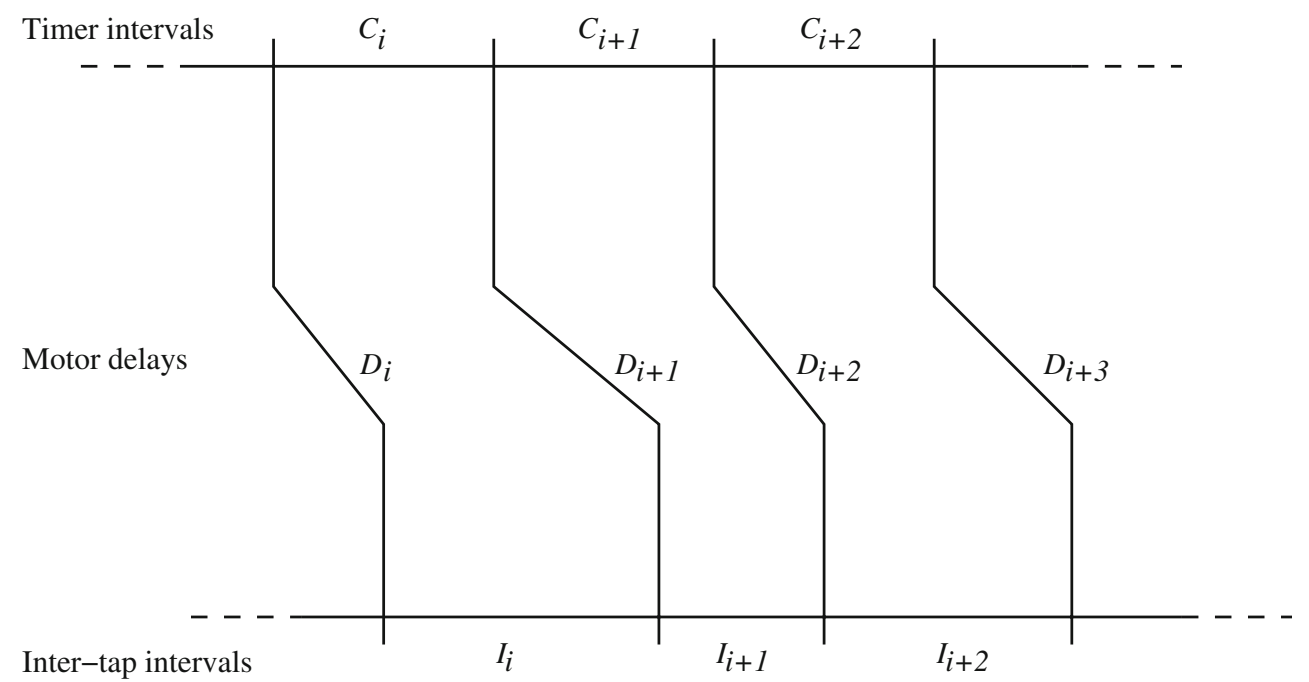

ITI is defined by $I_{i}=C_{i}+D_{i+1}-D_{i}$. Then, the ITI variability is determined by only two parameters: the clock interval variance $\sigma_{\mathrm{C}}^{2}$ and the motor delay variance $\sigma_{\mathrm{D}}^{2}$. These parameters can, in turn, be estimated using the autocovariance of the ITI sequences at lag zero and one: $\sigma_{D}^{2}=-\gamma(1)$ and $\sigma_{\mathrm{C}}^{2}=\gamma(0)+2 \gamma(1)$, in which $\gamma(k)$ denotes the ITI autocovariance at lag $k$. These forms can be combined as

$-0.5 \leq\left[\frac{\gamma(1)}{\gamma(0)}=\frac{-\sigma_{\mathrm{D}}^{2}}{\sigma_{\mathrm{C}}^{2}+2 \sigma_{\mathrm{D}}^{2}}\right] \leq 0$,

indicating that the lag-one serial autocorrelation of the ITIs is negative within the interval $[-0.5,0]$, i.e., errors in the ITIs will, on average, be compensated for in the following interval despite the absence of feedback.

The variability of the clock intervals has been found to increase monotonically with increasing interval duration (or decreasing movement frequency) (Vorberg and Wing 1996), implying that the ITI variance, $\gamma(0)=\sigma_{\mathrm{C}}^{2}+2 \sigma_{\mathrm{D}}^{2}$, increases as well. Note that the motor delay variance has been found to be constant over different tapping frequencies (Wing and Kristofferson 1973; Vorberg and Wing 1996). Hence, the absolute value of the lag-one autocorrelation will decrease with decreasing movement frequency. By using a tapping sequence that involves intentional drift, however, one may probe the clock in a different way than by considering different constant frequencies. By analyzing continuation tapping in this setting, we can examine the generality of the WK-model.

It is conceivable that the WK-model may account for tapping with intentional drift by simply recasting the clock interval as $C_{i}=C_{i}^{(0)}+\delta \cdot i$, where $C_{i}^{(0)}$ denotes the base clock interval and $\delta$ denotes a finite rate of change in clock interval. In this case, the autocorrelations will match those predicted by the WK-model. If, on the other hand, additional planning or conscious control is required, this and the added mental load of the drift may cause the autocorrelations to diverge from the WK-model predictions. As explained above, a monotonic drift cannot be maintained for an extended period of time simply due to neuromechanical constraints; the closer one gets to one's maximal tapping speed, the more attenuated the drift will become, and eventually the increase in tempo will saturate. Such ceiling effects on drift might also cause the autocorrelations calculated from intentional drift sequences to differ from those predicted by the WK-model.

After correcting for the predictable linear drift in tapping sequences, one is left with an isochronous sequence which can be analyzed in terms of autocorrelations and compared to the timer properties as predicted by the WKmodel for constant frequency sequences. Knowing that the autocorrelations found in this study matched those described by the WK-model, we used the WK-model to estimate the clock and motor delay variance. Obviously, in the case of intentional drift, the more non-linear the drift, the worse the estimates of these parameters, as will become apparent below.

\section{Experiment}

The experiment consisted of two separate sessions. During the first session, only behavioral signals, i.e., tapping events, were recorded, whereas during the second session surface EMG and magnetoencephalographic signals were recorded as well. Here we concentrate on the behavioral and EMG results; the neuroimaging data will be reported elsewhere.

In total, 21 healthy subjects without formal musical training participated in the experiment after signing an 
informed consent form, ten in the first session (five males and five females; age: mean 28.4, SD 4.0 years) and 11 (eight males and three females; age: mean 34.9, SD 8.6 years) in the second session. The experiment was approved by the local ethics committee. All subjects were right-handed according to their scores on the Edinburgh inventory (Oldfield 1971).

The experimental task was a synchronization-continuation tapping task with the right hand. The task was performed in four conditions; three constant frequency conditions $(1.5,2.0$, and $2.5 \mathrm{~Hz})$ and an intentional drift condition involving linearly decreasing ITIs corresponding to an increase in frequency from 1.5 to $2.5 \mathrm{~Hz}^{3}$ During the synchronization phase of the intentional drift condition the pacing of the ITIs decreased by $4 \mathrm{~ms}$ per tap. Subjects were asked to continue this decrease. The decrease of $4 \mathrm{~ms}$ per tap is noticeable at a starting ITI corresponding to $1.5 \mathrm{~Hz}$, as determined by Madison and Merker (2005). The task was performed for $35 \mathrm{~s}$ with $10 \mathrm{~s}$ of acoustic binaural pacing at the beginning (stimulus: pitch $440 \mathrm{~Hz}$, duration $50 \mathrm{~ms}$ ). Subjects were verbally informed of the end of each experimental trial. Each condition was presented as a block of six data collection trials. Before starting these trials, the subject practiced the desired frequency or drift by following a completely paced sequence. Halfway through the six data collection trials, the subjects were given $35 \mathrm{~s}$ of rest. Each of the four conditions (as a block of six trials) was performed twice, and the order of the frequencies was counterbalanced. Both sets of the intentional drift condition were performed either before or after the constant frequency conditions. This order (before or after) was randomized. The total experiment for each session thus consisted of eight blocks of six data collection trials each.

The protocol was implemented in Labview (National Instruments, Austin, TX, USA). Subjects were seated upright in a comfortable position, with their hands and arms supported by arm rests. Both hands lay flat on the arm rests and the force produced by taps of the right index finger was recorded using a force transducer (sampling rate: $1 \mathrm{kHz}$ for the first set, and $1.25 \mathrm{kHz}$ for the second data set). For the second data set, EMG was recorded from the flexor and extensor of the right index finger $\left(\mathrm{EMG}_{\mathrm{flex}}\right.$ : m. flexor digitorum, $\mathrm{EMG}_{\mathrm{ext}}:$ m. extensor digitorum) where the ground electrode was positioned on the lateral epicondyle of the right humerus.

\footnotetext{
3 Pilot studies revealed that subjects had great difficulty increasing their ITIs in a regular, linear fashion, often resulting in a marked decrease in tempo followed by a very slow tapping frequency. Therefore, drift with a decreasing frequency was deemed unsuitable for this study.
}

\section{Data analysis}

The tap events were determined from the force response of the force transducer. Figure 2 shows a typical force response. The tap event was defined as the start of the sharp increase prior to the force peak. The event detection was minimally influenced by noise, yielding an error of a couple of samples (i.e., a few ms) at most. Only complete tapping sequences, displaying separate force profiles for each tap and without 'misses' (992 out of 1,008) were included. For all analyses based on tap events, the continuation phase was used starting $14 \mathrm{~s}$ after the start of a trial to avoid adverse effects from the synchronization to continuation transition. For the intentional drift condition this implies that the starting frequency was $1.7 \mathrm{~Hz}$ and that the average ITI was $400 \mathrm{~ms}$ corresponding to a frequency of $2.0 \mathrm{~Hz}$. From the force profiles, the amount of force per tap was calculated as the area underneath the force profile. ITIs were defined as the time difference between two successive tap events. Figure 3 shows the ITIs of a trial in the intentional drift condition. Autocorrelation functions for 947 (see below) ITI sequences were computed for trials with a continuation sequence of at least 30 events. Autocorrelograms of the ITI sequences, covering lags 0 through 7 , were calculated using the 30 consecutive taps of each trial that showed the least drift (i.e., where the slope of the linear fit was closest to zero for the constant frequency conditions), and 30 points for each drift trial that showed

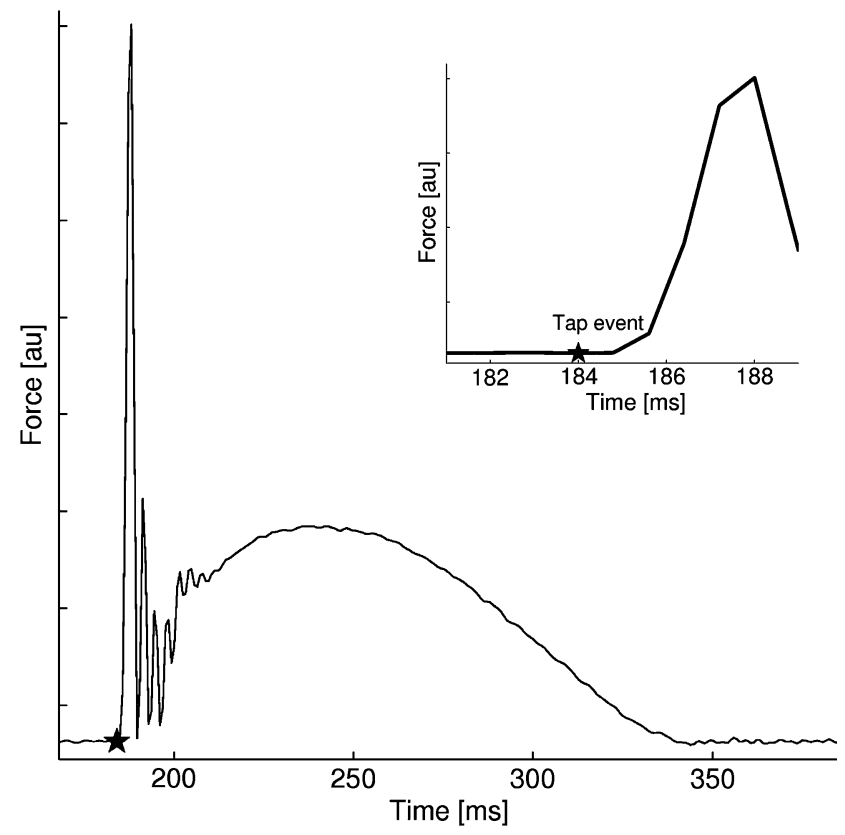

Fig. 2 Typical force profile, with force displayed in arbitrary units (au). The tap event was defined as the start of the sharp increase prior to the force peak as indicated by the asterisk. The inset shows a more detailed view of the same force profile at the time-point of event detection (marked with an asterisk) 


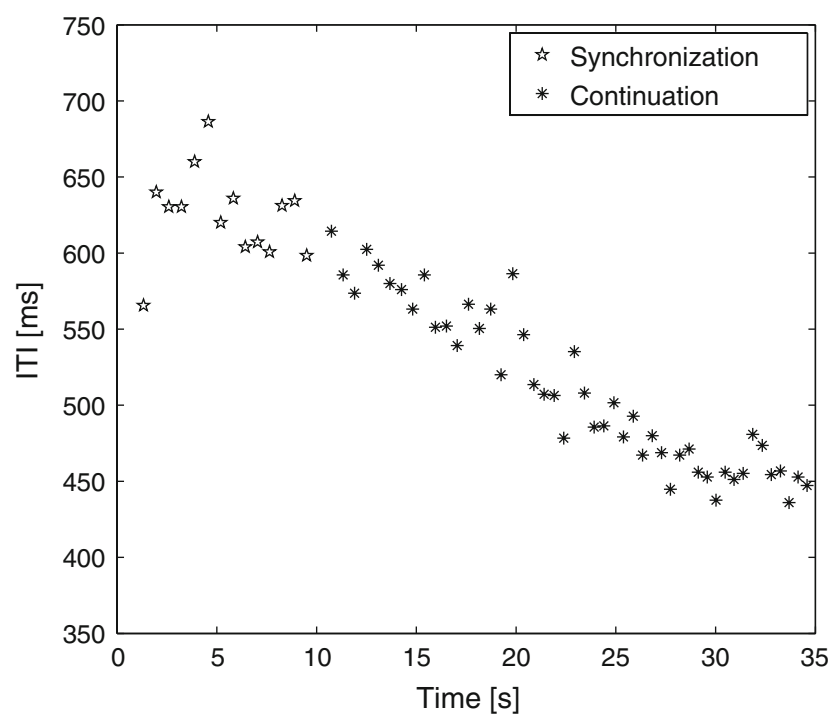

Fig. 3 ITIs during an intentional drift sequence

the smallest deviation from linear drift (in the intentional drift condition). This procedure was followed to eliminate the error in the autocorrelation estimates due to non-linear drift as much as possible. Drift sequences were only included if they showed a total decrease of at least $0.22 \mathrm{~Hz}$ (approximately 25\% of the target drift) and did not exceed $4 \mathrm{~Hz}$ (approximately three times the target drift). In total, 45 sequences were discarded. The resulting 947 sequences were linearly detrended before computing autocorrelations (Doumas and Wing 2007).

To compare the degree of difficulty of the intentional drift condition to that of the constant frequency conditions, we compared performance errors, in terms of the deviation from the intended ITIs, of the entire continuation part of all 947 sequences. We compared the target change in interval length $(0 \mathrm{~ms} / \mathrm{tap}$ for the constant frequency conditions, $-4 \mathrm{~ms} / \mathrm{tap}$ for the intentional drift condition) to the actual change. For artifact removal the EMGs were band-pass filtered between 10 and $400 \mathrm{~Hz}$ (second order bi-directional Butterworth). Signals were subsequently used to estimate the physical delay (PD), i.e., the time between muscle activation onsets and tap events, which covers the mechanical aspect of the motor delay in the WK-model. EMG onsets were derived with a generalized likelihood ratio estimator (Micera et al. 1998; Stylianou et al. 2003), using a test function based on the linear envelope of the EMGs. The EMG traces were full-wave rectified to obtain a linear envelope (Myers et al. 2003). For each trial, these EMG traces were averaged over tap events to increase the signal-to-noise ratio. PD values were determined for each trial, and mean and variance values were calculated for each participant and conditions based on these data. The values averaged over participants are given in Table 3 as discussed below. A total of 142 trials (out of 512) in which the PD could not be determined within a range of 150 $50 \mathrm{~ms}$ prior to the tap were discarded. In addition to PD, we quantified the co-activation of the flexor and extensor muscles via a co-contraction index (CCI), which was calculated for the full-wave rectified EMG traces during the continuation phase as

$$
\mathrm{CCI}=\frac{\overline{\mathrm{EMG}_{\text {flex }} \cdot \mathrm{EMG}_{\mathrm{ext}}}}{\overline{\mathrm{EMG}_{\mathrm{flex}}} \cdot \overline{\mathrm{EMG}_{\mathrm{ext}}}}
$$

where $\cdots$ denotes the mean over time.

Statistics

To assess the influence of frequency, one-way repeated measures analyses of variance (ANOVAs) were conducted per outcome variable on the constant frequency conditions for which values were averaged over trials. A significance level of $\alpha=0.05$ was maintained. The design, given a medium effect-size $\left(\omega^{2}=0.25\right)$, had a power of 0.73 for analyses involving 11 participants, and a power of 0.98 for analyses involving 21 participants. Error-bars in figures represent the standard error.

\section{Results}

The force data from both sessions were pooled. Performance accuracy was measured by the deviation from the intended change in ITI $(0 \mathrm{~ms} / \mathrm{tap}$ for the constant frequency conditions, $-4 \mathrm{~ms} / \mathrm{tap}$ for the intentional drift condition, as shown in Fig. 4).

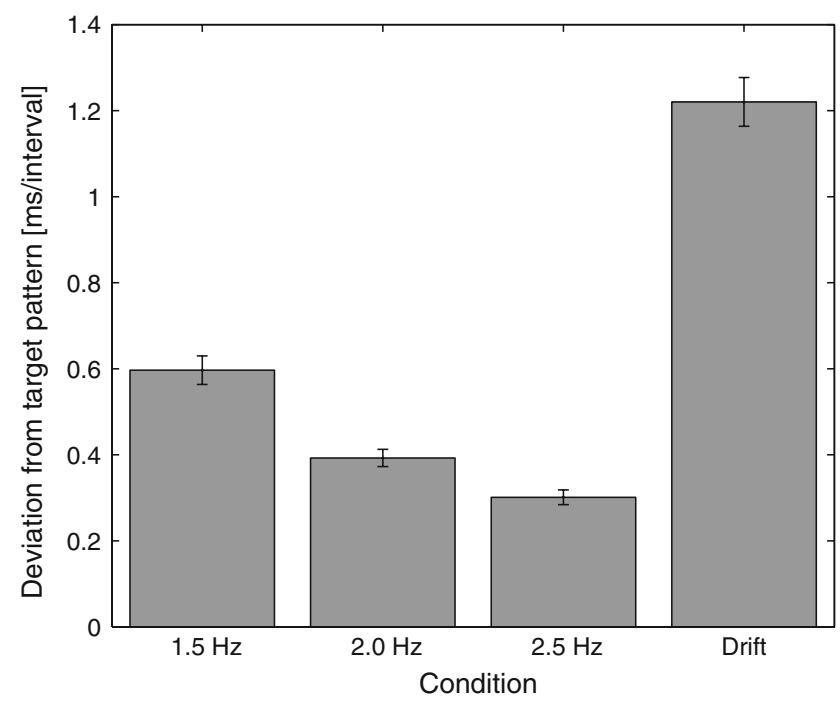

Fig. 4 Performance accuracy as measured by the deviation from the intended change in ITI 
There was a significant influence of tapping frequency on the performance accuracy of the constant frequency conditions $(F(2,40)=28.18, P<0.0001)$. As indicated in the "Introduction", the intentional drift condition was properly performed but significantly less well than any of the constant frequency conditions $(P s<0.0001)$. Intentional drift is thus considered to be a more challenging sequence to tap than a constant tempo. Tap force profiles became shorter as tapping frequency increased and the total force per tap decreased. This significant change in force level was confirmed $(F(2,40)=10.41, P<0.001)$ and mean values are summarized in Table 1 . All pairs were significantly different from each other $(P s<0.05)$.

Recall that the ITI variance is known to increase with increasing target interval (e.g., Vorberg and Wing 1996). Figure 5 shows the ITI variance for each condition. A significant effect of tapping frequency on ITI variance in the constant frequency conditions was found $(F(2,40)=53.09, P<0.001)$. Post hoc tests revealed that all pairs were significantly different from each other $(P s<0.05)$. In the drift condition, this trend was also present. To demonstrate this, the drift data were split into two sets of 15 events each. As expected, and consistent with the trend found in the ITI variance of the constant

Table 1 Force levels, residuals of linear fit to ITI sequences $[$ mean \pm std. error, in arbitrary units (au)]

\begin{tabular}{llc}
\hline Condition & Force (au) & Residuals (au) \\
\hline $1.5 \mathrm{~Hz}$ & $72 \pm 5$ & $126 \pm 31$ \\
$2.0 \mathrm{~Hz}$ & $54 \pm 4$ & $103 \pm 28$ \\
$2.5 \mathrm{~Hz}$ & $43 \pm 3$ & $86 \pm 23$ \\
Drift & $61 \pm 5$ & $93 \pm 22$ \\
\hline
\end{tabular}

conditions, the ITI variance of the slow part of the drift was significantly larger $(t(19)=1.99, P<0.05)$ than that of the fast part (see Fig. 5), although the difference was smaller than expected from the values found in the constant frequency conditions.

The 30 events that showed the most constant or most linear behavior were selected from each tapping sequence. The deviation from that constant value or from the linear drift could thus be used to compare the constant frequency conditions with the drift condition. We used the residuals of the linear fit to the data to assess this. Table 1 displays the means and standard errors of the residuals for each condition. A significant influence of tapping frequency on the size of the residuals was found in the constant frequency conditions $(F(2,40)=73.29, P<0.001)$. All pairs were significantly different from each other $(P s<0.05)$. The residuals of the intentional drift condition fell between those of the 2.0 and $2.5 \mathrm{~Hz}$ condition. This was expected as the average ITI during the continuation phase of the intentional drift condition corresponded to a frequency of $2.0 \mathrm{~Hz}$, but was often slightly higher. The variance is also similar to the 2.0 and $2.5 \mathrm{~Hz}$ conditions (see Fig. 5). The goodness-of-fit showed a similar trend to that found for the ITI variability (see also Table 1).

Autocorrelograms of the analyzed tapping sequences are shown in Fig. 6. All constant frequency conditions showed a clear negative lag-one serial autocorrelation and autocorrelation values close to zero for higher lags. Interestingly, the intentional drift condition also showed a clear negative lag-one autocorrelation and small negative values for lags 2 through 4 . Larger negative values were found for lags 5-7. Table 2 summarizes the results of onesample $t$ tests performed for each condition for each lag. Significant results at lags 5 through 7 in the intentional drift
Fig. 5 ITI variance in the three constant frequency conditions and the drift analyzed in a slow and a fast part
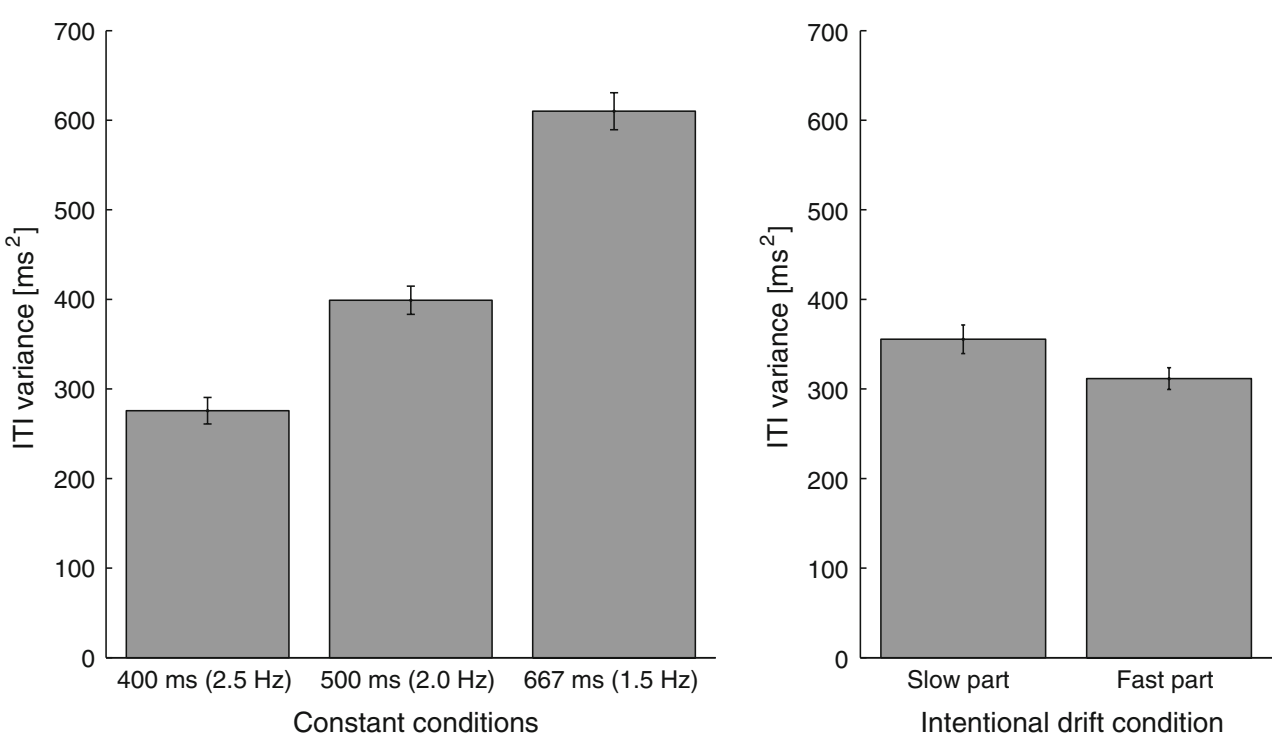
Fig. 6 Autocorrelation function (lag 1-7) for each condition averaged over trials and subjects
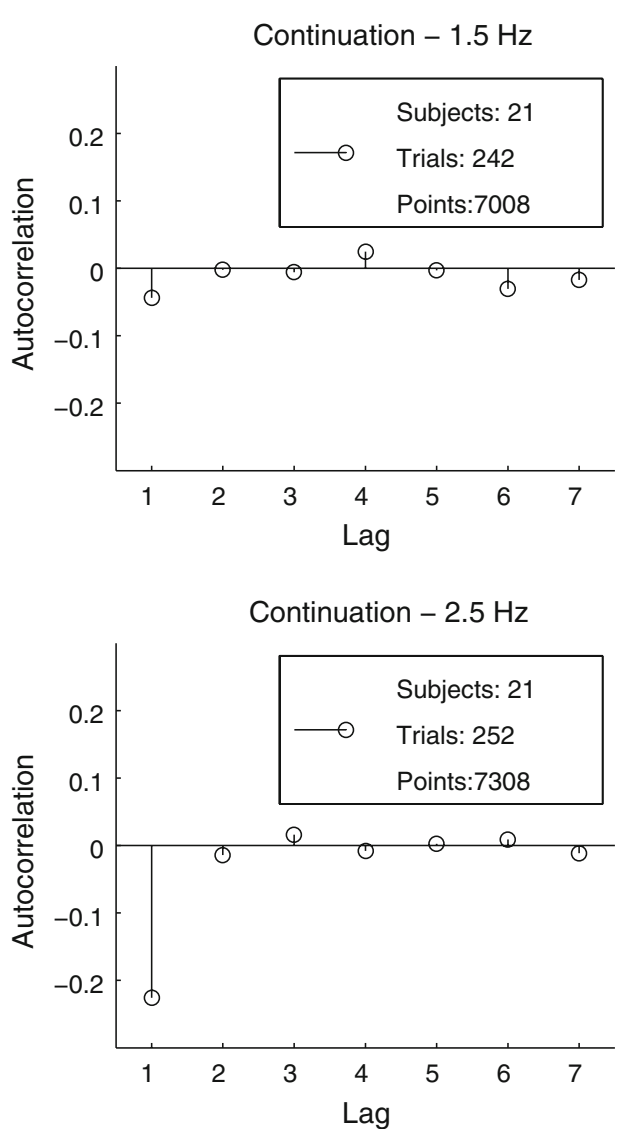

Continuation $-2.0 \mathrm{~Hz}$

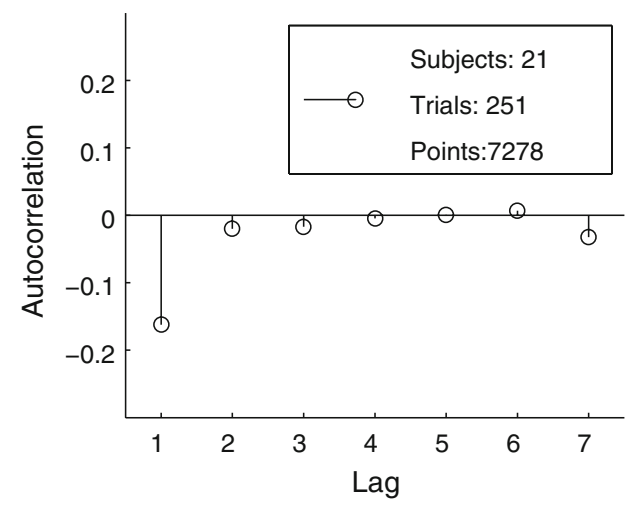

Continuation - Intentional drift

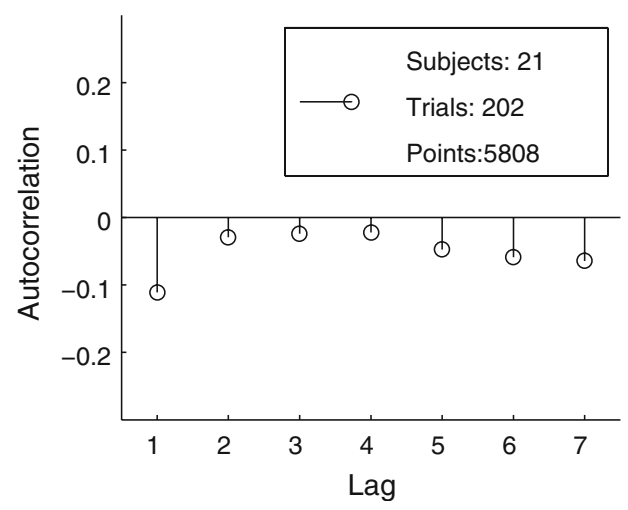

Table 2 One-sample $t$ test $P$ values for each condition and autocorrelation lag

\begin{tabular}{|c|c|c|c|c|c|c|c|}
\hline \multirow[t]{2}{*}{ Condition } & \multicolumn{7}{|l|}{ Lag } \\
\hline & 1 & 2 & 3 & 4 & 5 & 6 & 7 \\
\hline $1.5 \mathrm{~Hz}$ & $<0.001$ & 0.164 & 0.806 & $<0.05$ & 0.785 & 0.100 & 0.120 \\
\hline $2.0 \mathrm{~Hz}$ & $<0.0001$ & 0.583 & 0.671 & 0.897 & 0.834 & 0.554 & $<0.001$ \\
\hline $2.5 \mathrm{~Hz}$ & $<0.0001$ & 0.259 & 0.125 & 0.377 & 0.762 & 0.215 & 0.280 \\
\hline Drift & $<0.0001$ & 0.231 & 0.071 & 0.542 & $<0.01$ & $<0.01$ & $<0.001$ \\
\hline
\end{tabular}

Significant results are in bold

condition reflected non-linearities in the drift, where the sign and the decreasing values of the autocorrelations at these lags were consistent with a saturation of the drift. ${ }^{4}$ Nonetheless, the structures of the autocorrelations were comparable across conditions, where the negative lag-one autocorrelation for the intentional drift condition fell between those for the 1.5 and $2.0 \mathrm{~Hz}$ conditions.

As described earlier, we decomposed the ITI variance into clock variance and motor delay variance. In the WK-model, only the clock variance is supposed to be

\footnotetext{
${ }^{4}$ In the case of saturating ITIs, the detrending will result in later ITIs being smaller than the first ones. Therefore, saturation yields negative autocorrelations and this effect is more pronounced for higher lags.
}

frequency dependent. However, Doumas and Wing (2007) found a small but statistically significant decrease in motor delay SD for smaller ITIs. In our study, this decrease, although present in the motor delay variance, failed to reach significance $(F(2,40)=3.11, P=0.055)$. The effect of frequency on clock variance was significant $(F(2,40)=44.66, P<0.001)$ and all pairs were significantly different from each other $(P s<0.05)$. Figure 7 (panels A1 and A2) shows the values of the model parameter estimates. The influence of tapping frequency on the clock variance estimate was much greater than on the motor delay variance estimate.

The model parameter estimates of some sequences fell outside the boundaries mentioned in the section on the WK-model, that is, the lag-one autocorrelation fell outside the interval $[-0.5,0]$. This was the case for 254 of the 992 trials. When removing those sequences from the analysis (the results for the remaining sequences are shown in Fig. 7, panels B1 and B2), we found similar results for the timer variance $(F(2,40)=32.48, P<0.001)$. Post hoc test revealed that all pairs were significantly different from each other $(P s<0.05)$. In addition, we found a significant influence of tapping frequency on the motor delay variance, where, in line with Doumas and Wing (2007), shorter tap intervals had a smaller value for $\sigma_{\mathrm{D}}^{2}(F(2,40)=5.20$, $P<0.05)$. The motor delay variance at $1.5 \mathrm{~Hz}$ was 
Fig. 7 Model parameter estimates per condition. Timer and motor delay variance when all sequences are considered (panels $A 1$ and $A 2$ ), and when only sequences are considered that adhere to the WK-model boundaries (panels $B 1$ and $B 2$ )
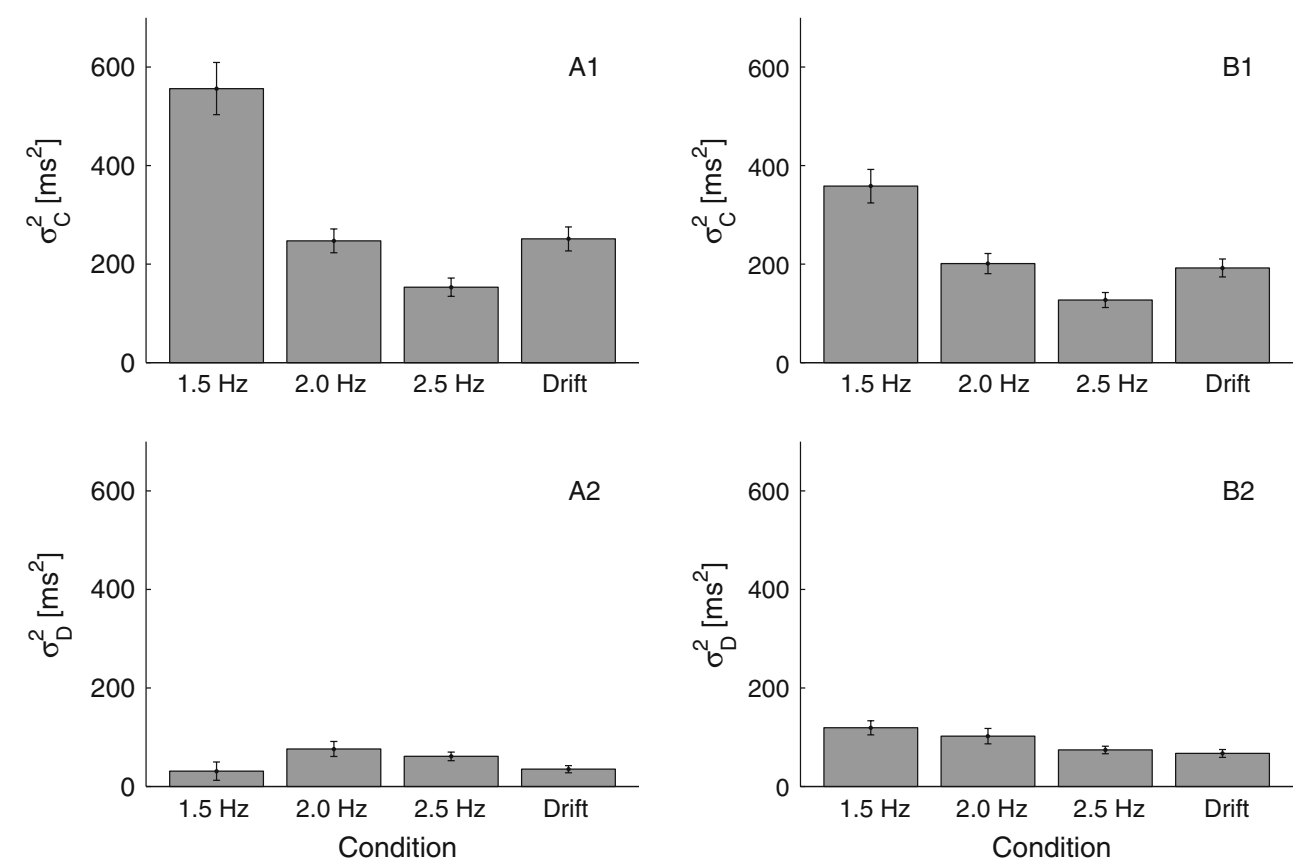

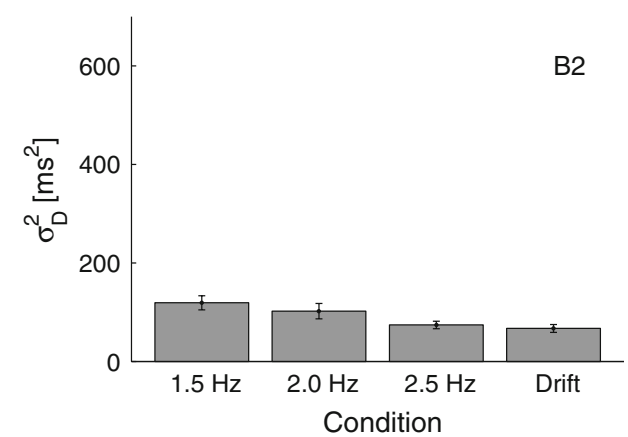

significantly larger than at $2.5 \mathrm{~Hz}$, and the motor delay variance at $2.0 \mathrm{~Hz}$ was larger than at $2.5 \mathrm{~Hz}(P s<0.05)$.

To examine the influence of tapping frequency on motor variance we used the $\mathrm{EMG}_{\text {flex }}$ signals. One can assume that the motor delay has a neural and a mechanical component. In view of the brevity of the neural delay between primary motor areas and proximal muscles, in particular the finger flexors (Nezu et al. 1999), we assume that the larger part of the motor delay will be the time from neural activation of the muscles to the actual tap, including the movement time of the index finger. As the amount of force per tap is dependent on tapping frequency, the PD may also depend on tapping frequency. A significant influence of tapping frequency in the constant conditions on PD was found $(F(1.10,7.77)=6.841, \quad P<0.05, \quad$ Greenhouse-Geisser correction). In contrast, there was no significant influence on PD variance $(F(1.04,7.3)=0.39, P=0.559$, Greenhouse-Geisser correction), although there was a trend in that the PD values tended to increase. PD and PD variance values are given in Table 3. PD during intentional drift was comparable to levels found in the constant frequency conditions. There was no clear relation between tapping frequency and PD variance.

Table 3 Physical delay (PD) mean and variance

\begin{tabular}{lcl}
\hline Condition & $\mathrm{PD}_{\text {mean }}(\mathrm{ms})$ & $\mathrm{PD}_{\text {variance }}\left(\mathrm{ms}^{2}\right)$ \\
\hline $1.5 \mathrm{~Hz}$ & 92 & 585 \\
$2.0 \mathrm{~Hz}$ & 113 & 334 \\
$2.5 \mathrm{~Hz}$ & 119 & 428 \\
Drift & 102 & 463 \\
\hline
\end{tabular}

We found a significant influence of tapping frequency on CCI $(F(2,20)=8.77, P<0.005)$, indicating a reduction of co-activation of the flexor and extensor muscles at higher tapping frequencies (Fig. 8). CCI was larger at $1.5 \mathrm{~Hz}$ than at both $2.0 \mathrm{~Hz}$ and $2.5 \mathrm{~Hz}(P s<0.05)$. Muscle co-activation during intentional drift was comparable to levels found in the constant frequency conditions.

\section{Discussion and conclusion}

Here, we studied rhythmic tapping with and without intentional drift to examine the generality of the WK-

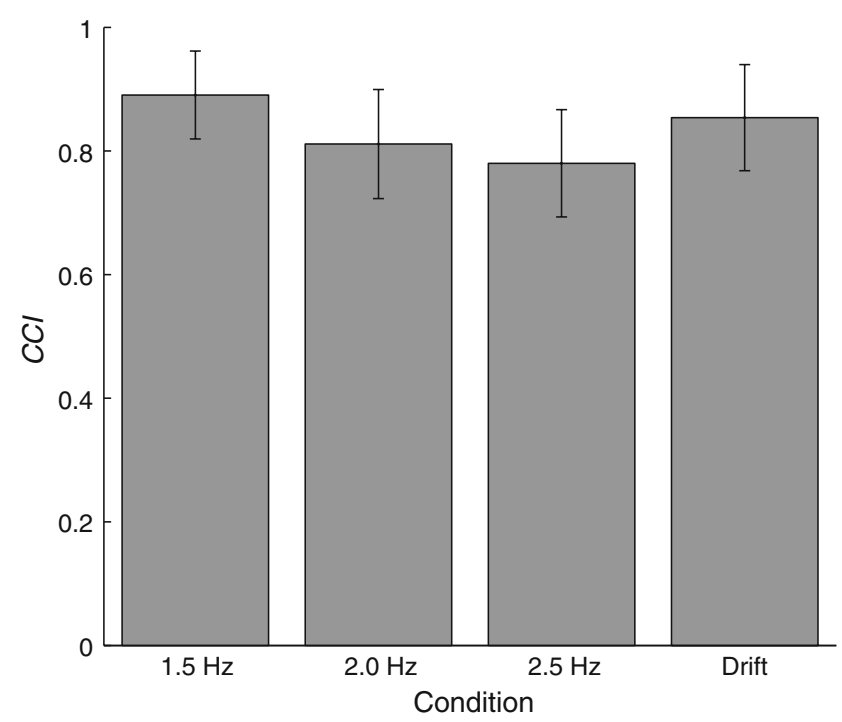

Fig. 8 CCI for each condition 
model and properties of neural timing in general. For this purpose, we compared parameter estimates of the WKmodel and additional measures (force, lag-one serial autocorrelation, ITI variability, PD, and muscle co-activation) across experimental conditions. We were especially interested in the comparison between tapping at a constant frequency and tapping at a continuously increasing frequency because the time base of the neural clock may be assumed to be fixed in the former case and must be continuously adjusted in the latter. In this light, it was not surprising that subjects reported the intentional drift condition to be much more difficult to perform than tapping a constant tempo. In line, and as expected on the basis of a pilot study, we found that performance was less accurate for the intentional drift condition. However, in spite of these differences between tasks, we found that most variables, after compensating for the drift by linear detrending, were similar, if not identical, for tapping with intentional drift and tapping at a constant tempo. This general result implies that the basic partitioning of continuation tapping into a clock interval and a motor delay, which is inherent to the WK-model, does not only describe isochronous tapping but also adequately describes tapping linearly decreasing intervals. Moreover, the temporal properties of the underlying timing processes are similar across conditions.

Task difficulty in terms of motor planning, control, and mental load might have influenced the temporal properties, as reflected in the autocorrelations, of the timer responsible for producing the tapping sequences. We have established that the intentional drift task was performed less well than constant tapping. However, since temporal properties matched across conditions, we can conclude that (experienced) task difficulty is not manifested in the timing of motor performance. We therefore conclude that, as the WK-model adequately describes the intentional drift task, the decomposition of ITIs into a clock interval and a motor delay as implied by the WK-model is properly extended to this task as well. We may even speculate that this result may be extended to non-linear (largely) deterministic drifts. Equivalent to the linear case, the model's clock intervals may be replaced by $C_{i}=C_{i}^{(0)}+\delta(i)$ with $\delta(i)$ denoting an arbitrary function that depends on the interval number $i$, i.e., on time. Subtracting that function from the ITI sequence, that is, detrending the ITIs, will recover the negative lag-one serial correlation as implied by the WKmodel. ${ }^{5}$

However, a number of properties warrant further discussion. The negative serial lag-one autocorrelation was closer to zero than expected during intentional drift. The most likely explanation of this finding is that subjects did

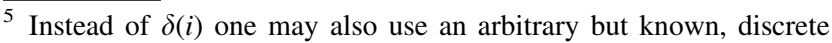
sequence $\delta_{i}$.
}

not perform a purely linear drift. There is evidence that drift perception is governed by a detection threshold for a change in tempo (e.g., Madison 2001). If drift production involves a similar process, drift would show abrupt changes and would not be linear and monotonic. As a consequence, the detrending procedure applied here would have been insufficient to compensate for the ensuing nonlinearities in the produced drift. Madison (2001) showed that this kind of influence results in lag-one autocorrelation estimates closer to zero (i.e., less negative than the WKmodel predicts). Despite the deviations from linearity and the fact that drift can be modeled in a variety of ways (Madison 2001; Collier and Ogden 2004), the linear fit found here was well within the range of the constant frequency conditions, implying that the subjects followed the instructions to a satisfactory degree.

Irrespective of the lower performance accuracy (in terms of the deviation from the intended change in ITI) during the intentional drift condition due to the greater difficulty of this pattern, the ITIs used in the analysis did not show higher variability than that found in the constant frequency conditions. In contrast, Doumas and Wing (2007) found a higher variability for a more difficult task (tapping a rhythm), indicating that tapping with an intentional drift is more similar to tapping at a constant pace than tapping a particular rhythm. It is possible that, although there is a need for more involved planning in this task, movement execution does not interact with this planning. In that case, any perceived difficulty due to planning would not transfer to tapping behavior. A recent study by Lewis et al. (2004) supports this interpretation as initiation, synchronization, and continuation were found to be affected in different ways during the performance of tasks with different degrees of difficulty. On the other hand, because our subjects had no musical training, it is possible that they were not able to make on-line corrections during their performance. By asking well-trained musicians to perform the same task, it might be possible to gain insight into this matter.

Besides broadening the class of tapping behaviors described by the WK-model, the present study produced several insights into the clock and motor aspects of isochronous and non-isochronous tapping, which we discuss in turn, starting with the motor aspects. An unresolved issue with regard to the motor delays is whether their variance depends on the tapping frequency. Whereas the WK-model assumes that this is not so, a recent study reported a small but significant dependence (Doumas and Wing 2007). In the present study, we found a similar dependence, at least when the analysis was confined to tapping sequences with lag-one autocorrelations within the interval $[-0.5,0]$ predicted by the WK-model. Given that the WK-model only provides an estimate of the variance of 
the motor delay, we attempted to estimate part of the motor delay by analyzing the PD and its variance, but with inconclusive results. We also analyzed the level of muscle co-activation and found a significant influence of tapping frequency on CCI. In addition, like Sternad et al. (2000), we found that the amount of force per tap was smaller for higher tapping frequencies. All in all, the evidence for an influence of tapping frequency on PD appears mixed and, as it stands, inconclusive. Yet, considering that muscletendon complexes have non-linear, time-dependent relationships (Spanjaard et al. 2007, in press), and that movement trajectories depend on tapping frequency (Doumas and Wing 2007), we believe it is likely that motor delays and their variance do depend on tapping frequency, at least to a degree.

As regards the clock aspects, the WK-model does not address the neural organization of the timer. It is difficult, if not impossible, to gain insight into this aspect solely from behavioral data (Wing 2002). From imaging studies we know that multiple brain areas are involved in motor timing tasks in a task-dependent fashion (Turner et al. 2003; Mayville et al. 2005). In view of the discussed differences between constant frequency tapping and tapping with intentional drift, it is therefore rather likely that different networks were involved in both forms of tapping, potentially controlling repetitive movements with different temporal properties. Hence, we find it remarkable that the behavioral properties of interest were so similar between isochronous and non-isochronous tapping. This finding speaks for the existence of an, abstractly defined, neural timer with invariant characteristics that are preserved over different task instantiations. Whether this is indeed the case remains to be established because, in principle, it is conceivable that differences in neural assembly processes are washed out when transferred to motor output. To resolve this issue it is necessary to study the connection between brain activity and motor output, including the total delay between the central timer and the motor output, as well as their variances. A promising approach might be an assessment of neural activation with high temporal resolution, e.g., with encephalographic recordings, with an additional source localization to estimate activities in subcortical areas, basal ganglia, the cerebellum, etc., in conjunction with detailed motor output recordings. We are currently conducting investigations along these lines.

Acknowledgments We would like to thank the Dutch Science Foundation for financial support (NWO grant \# 452-04-344).

Open Access This article is distributed under the terms of the Creative Commons Attribution Noncommercial License which permits any noncommercial use, distribution, and reproduction in any medium, provided the original author(s) and source are credited.

\section{References}

Chen Y, Ding M, Kelso JAS (1997) Long memory processes (1/f alpha type) in human coordination. Phys Rev Lett 79:4501

Collier GL, Ogden RT (2004) Adding drift to the decomposition of simple isochronous tapping: an extension of the Wing-Kristofferson model. J Exp Psychol Hum Percept Perform 30:853-872

Daffertshofer A (1998) Effects of noise on the phase dynamics of nonlinear oscillators. Phys Rev E 58:327

Del Olmo MF, Cheeran B, Koch G, Rothwell JC (2007) Role of the cerebellum in externally paced rhythmic finger movements. $\mathrm{J}$ Neurophysiol 98:145-152

Delignières D, Lemoine L, Torre K (2004) Time intervals production in tapping and oscillatory motion. Hum Mov Sci 23:87-103

Dhamala M, Pagnoni G, Wiesenfeld K, Berns GS (2002) Measurements of brain activity complexity for varying mental loads. Phys Rev E 65:041917

Ding M, Chen Y, Kelso JAS (2002) Statistical analysis of timing errors. Brain Cogn 48:98-106

Doumas M, Wing AM (2007) Timing and trajectory in rhythm production. J Exp Psychol Hum Percept Perform 33:442-455

Gerloff C, Corwell B, Chen R, Hallett M, Cohen LG (1998) The role of the human motor cortex in the control of complex and simple finger movement sequences. Brain 121(Pt 9):1695-1709

Grahn JA, Brett M (2007) Rhythm and beat perception in motor areas of the brain. J Cogn Neurosci 19:893-906

Ivry RB (1997) Cerebellar timing systems. Int Rev Neurobiol 41:555573

Ivry RB, Keele SW, Diener HC (1988) Dissociation of the lateral and medial cerebellum in movement timing and movement execution. Exp Brain Res 73:167-180

Ivry RB, Richardson TC (2002) Temporal control and coordination: the multiple timer model. Brain Cogn 48:117-132

Kaulakys B (1999) Autoregressive model of 1/f noise. Phys Lett A 257:37-42

Lewis PA, Wing AM, Pope PA, Praamstra P, Miall RC (2004) Brain activity correlates differentially with increasing temporal complexity of rhythms during initialisation, synchronisation, and continuation phases of paced finger tapping. Neuropsychology 42:1301-1312

Loehr JD, Palmer C (2007) Cognitive and biomechanical influences in pianists' finger tapping. Exp Brain Res 178:518-528

Madison G (2001) Variability in isochronous tapping: higher order dependencies as a function of intertap interval. J Exp Psychol Hum Percept Perform 27:411-422

Madison G, Merker B (2005) Timing of action during and after synchronization with linearly changing intervals. Music Percept 22:441-459

Manganotti P, Gerloff C, Toro C, Katsuta H, Sadato N, Zhuang P, Leocani L, Hallett M (1998) Task-related coherence and taskrelated spectral power changes during sequential finger movements. Electroencephalogr Clin Neurophysiol 109:50-62

Mayville JM, Fuchs A, Kelso JAS (2005) Neuromagnetic motor fields accompanying self-paced rhythmic finger movement at different rates. Exp Brain Res 166:190-199

Micera S, Sabatini AM, Dario P (1998) An algorithm for detecting the onset of muscle contraction by EMG signal processing. Med Eng Phys 20:211-215

Musha T, Katsurai K, Teramachi Y (1985) Fluctuations of human tapping intervals. IEEE Trans Biomed Eng 32:578-582

Myers LJ, Lowery M, O’Malley M, Vaughan CL, Heneghan C, St Clair Gibson A, Harley YX, Sreenivasan R (2003) Rectification and non-linear pre-processing of EMG signals for corticomuscular analysis. J Neurosci Meth 124:157-165 
Nezu A, Kimura S, Takeshita S (1999) Topographical differences in the developmental profile of central motor conduction time. Clin Neurophysiol 110:1646-1649

Oldfield RC (1971) The assessment and analysis of handedness: the Edinburgh inventory. Neuropsychol 9:97-113

Pressing J (1998) Error correction processes in temporal pattern production. J Math Psychol 42:63-101

Pressing J, Jolley-Rogers G (1997) Spectral properties of human cognition and skill. Biol Cybern 76:339-347

Schulze H-H, Cordes A, Vorberg D (2005) Keeping synchrony while tempo changes: accelerando and riterdando. Music Percept 22:461-477

Spanjaard M, Reeves N, van Dieën J, Baltzopoulos V, Maganaris C (2007) Influence of gait velocity on gastrocnemius muscle fascicle behaviour during stair negotiation. J Electromyogr Kinesiol (in press)
Sternad D, Dean WJ, Newell KM (2000) Force and timing variability in rhythmic unimanual tapping. J Mot Behav 32:249-267

Stevens LT (1886) On the time sense. Mind 11:393-404

Stylianou AP, Luchies CW, Insana MF (2003) EMG onset detection using the maximum likelihood method. In: Proceedings of the 2003 summer bioengineering conference, pp 1075-1076

Turner RS, Desmurget M, Grethe J, Crutcher MD, Grafton ST (2003) Motor subcircuits mediating the control of movement extent and speed. J Neurophysiol 90:3958-3966

Vorberg D, Wing AM (1996) Modeling variability and dependence in timing. In: Heuer H, Keele SW (eds) Handbook of perception and action, vol 3. Academic Press, London, pp 287-299

Wing AM (2002) Voluntary timing and brain function: an information processing approach. Brain Cogn 48:7-30

Wing AM, Kristofferson B (1973) The timing of interresponse intervals. Percept Psychophys 3:455-460 